\title{
Wettbewerb im deutschen Gesundheitswesen \\ Ideen und Interessen bei der Reform der gesetzlichen Krankenversicherung
}

Bereits 1992 wurden erstmalig Wettbewerbsregelungen in das System der gesetzlichen Krankenversicherung eingeführt. Dennoch sind sich Gesundheitsökonomen weitestgehend darüber einig, dass das deutsche Gesundheitswesen noch immer nicht als durchweg wettbewerbsorientiert bezeichnet werden kann. Eine bislang vernachlässigte Ursache dafür sind unterschiedliche parteipolitische Überzeugungen hinsichtlich der Ausgestaltung dieses Wettbewerbs.

\section{Markus Grunenberg}

\section{Einleitung}

Mit seiner Vielzahl von Akteuren und seinen unterschiedlichen Regulierungsmechanismen gilt das deutsche Gesundheitswesen als besonders schwierig politisch steuerbar. Dies ist das Resultat sektoral getrennter Versorgungsstrukturen, in denen jeweils ein unterschiedliches Verhältnis von staatlichen, korporatistischen und wettbewerblichen Steuerungsinstrumenten herrscht. Erschwerend kommen die institutionellen Besonderheiten der deutschen Verhandlungsdemokratie hinzu. Die Bildung von Koalitionsregierungen, der Einfluss der Länder im Bundesrat und die Einbindung von Interessenverbänden machen Verhandlungslösungen oftmals notwendig, während diese gleichzeitig vom Parteienwettbewerb konterkariert werden. Hinsichtlich politischen Wandels wird deshalb angenommen, dass Veränderungen im deutschen Gesundheitswesen nur inkrementell möglich sind.

Trotz dieser Schwierigkeiten wird von der Politik der „große Wurf“ erwartet. Im Jahr 2006 verabschiedete die Große Koalition nach einem anstrengenden politischen Tauziehen das GKV-Wettbewerbsstärkungsgesetz, das als eines der umstrittensten Reformvorhaben der gesetzlichen Krankenversicherung (GKV) gilt. Bereits während des Aushandlungsprozesses hagelte es laute Kritik von den Akteuren der Gesundheitspolitik. Auch wenn die im Vorfeld vielerorts geforderte grundlegende Finanzierungsreform ausblieb, wurden dennoch tiefgreifende Veränderungen

Markus Grunenberg, PRGS Unternehmensberatung für Politik\& Krisenmanagement, Berlin im deutschen Gesundheitswesen durchgesetzt. Das Gesetz bildet den vorläufigen Höhepunkt einer zunehmend wettbewerblichen Ausrichtung der gesetzlichen Krankenversicherung. In diesem inkrementellen Prozess wurden sukzessive Wettbewerbsinstrumente eingeführt, so dass mittlerweile von einem „Steuerungswirrwarr" gesprochen wird. Doch wie lässt sich die Vielzahl unterschiedlicher, teilweise widersprüchlicher Regelungen erklären?

Zur sukzessiven Ausweitung von Wettbewerbsregelungen hat ausschlaggebend die Überzeugung der Politik beigetragen, dass eine wettbewerbliche Steuerung des Gesundheitssystems einen besseren Outcome erzeugen würde, als es das bis dahin in weiten Teilen korporatistische Steuerungsmodell schaffte. So wurde der wettbewerbliche Umbau des deutschen Gesundheitswesens seit Anfang der 1990er Jahre beständig vorangetrieben. Trotz dieser Bemühungen kann noch auch heute nicht von einem vollständig auf Wettbewerb ausgerichteten Gesundheitssystem gesprochen werden. Auch heute ist die Zahl solcher Instrumente auf einen Krankenkassenwettbewerb und bestimmte Selektivvertragsregelungen insbesondere im ambulanten Sektor begrenzt. Deshalb besteht eine Reihe von Vorschlägen und Forderungen, wie der Wettbewerb in der GKV ausgeweitet werden könnte. Dabei reichen diese Vorschläge von einer Einführung von Wettbewerbselementen in ein korporatistisches System bis hin zu einer umfassenden Liberalisierung der Vertragsbeziehungen in der GKV. Mit diesen Lösungen werden allerdings unterschiedliche Vorstellungen über die konkrete Ausgestaltung dieses Wettbewerbs verfolgt. 


\section{THEMA}

Verglichen mit anderen Politikfeldern weist das Gesundheitswesen eine Vielzahl von Akteuren mit konträren Interessen auf, denen oftmals nicht unerhebliches Obstruktionspotential bei der Umsetzung von Reformen innewohnt. Gleichzeitig ist die Gesundheitspolitik sehr stark von unterschiedlichen Ideen besetzt, weil in dem von Unsicherheit geprägten Politikfeld die Wahrnehmung von Handlungserfordernissen stark von Überzeugungen beeinflusst wird. Wegen der spezifischen Kompromissnotwendigkeit finden politische Entscheidungen in der Gesundheitspolitik deshalb als ein „Muddling-through“ mit wechselnden Akteurskonstellationen und Einflusspotentialen statt. Somit sind es nicht nur widerstreitende Interessen sondern auch verschiedene Ideen, die zu einer Vielzahl unterschiedlicher Wettbewerbsregelungen führen.

\section{Parteipolitische Interessen in der Gesundheitspolitik}

In groben Zügen lassen sich Interessen im Gesundheitswesen auf die vier Dimensionen Finanzierbarkeit, Qualität, Solidarität und Wachstum reduzieren (Bandelow 2006). Mit der Finanzierbarkeit werden eine Stabilisierung der Gesundheitsausgaben und die Sicherung der Einnahmen beabsichtigt. Die Dimension Qualität stellt ein hohes Niveau der Gesundheitsversorgung in den Vordergrund. Das Ziel Solidarität umfasst die gleichwertige Versorgung der gesamten Bevölkerung unabhängig vom jeweiligen Einkommen. Die Dimension Wachstum verfolgt das Ziel einer Sicherung und Schaffung von Arbeitsplätzen sowie der Gewinnmaximierung in der Gesundheitswirtschaft. Angenommen wird, dass sich alle vier Dimensionen nicht gleichzeitig optimieren lassen, weil stets Zielkonflikte bestehen.

Während beispielsweise mit der Finanzierbarkeit die im Gesundheitssystem eingesetzten Ressourcen reduziert werden sollen, machen Qualität und Wachstum eine genau entgegengesetzte Erhöhung der Ressourcen erforderlich. Auch das Solidaritätsziel kann mit den übrigen Zielen kollidieren, wenn diese wiederum mit der Ausweitung privater Finanzierung oder einer Gliederung von GKV-Leistungen in Grund- und Zusatzleistungen erreicht werden sollen. Politische Entscheidungen bilden diese Zielkonflikte ab, indem - wenn auch meist lediglich implizit - eine Entscheidung für und wider bestimmte Ziele getroffen wird, die sich dann in einer gesetzlichen Regelung widerspiegelt.

Mit dem überproportionalen Ausgabenanstieg stehen schon seit den 1970er Jahren die Kostendämpfung und damit das Ziel der Finanzierbarkeit im Mittelpunkt der Parteiinteressen (Wasem 1999). Trotz aller politischen Bemühungen konnte das Gebot der Beitragssatzstabilität allerdings nicht eingehalten werden. Die Prognosen zur demographischen Entwicklung und dem medizinischtechnischen Fortschritt verschärfen in der Wahrnehmung der Politik die ungelösten Finanzierungsfragen, aus denen häufig Handlungszwänge abgeleitet werden.
Aus parteipolitischer Sicht geht es bei dieser Diskussion vor allem darum, welches der drei übrigen Ziele Qualität, Solidarität und Wachstum im Kontext des oben beschriebenen Zielkonflikts am ehesten aufgegeben werden kann, um die Finanzierbarkeit des Gesundheitswesens sicherzustellen. Während die Union am ehesten die Preisgabe der Solidarität präferiert, kann aus Sicht der SPD das Wachstumsziel vernachlässigt werden. Weil beide Parteien den Anspruch einer bürgerlichen Volkspartei verfolgen, versuchen sie dennoch möglichst viele der Ziele gleichzeitig zu erreichen, um einem Großteil ihrer Wählerschaft gerecht zu werden. Die kleineren Parteien im Bundestag befinden sich dagegen in der komfortableren Position, keinen breiten gesellschaftlichen Konsens abbilden zu müssen. So verfolgt die FDP vornehmlich das Ziel Wachstum und lediglich nachrangig das der Finanzierbarkeit. Die Linkspartei hingegen legt ihren Fokus auf Solidarität und Qualität, während Wachstum und Finanzierbarkeit keine oder nur eine nebensächliche Rolle spielen. Die Grünen schließlich weisen das unschärfste Profil hinsichtlich konkreter gesundheitspolitischer Ziele auf. Finanzierbarkeit und Qualität lassen sich am ehesten als zentrale parteipolitische Interessen identifizieren.

Zusammenfassend lässt sich sagen, dass alle Bundestagsparteien zumindest bis zu einem gewissen Grad das Ziel der Finanzierbarkeit verfolgen. Mit ihren Präferenzen bezüglich der anderen Ziele jedoch divergieren auch ihre Vorstellungen, wie dieses Finanzierungsziel erreicht werden soll. Mittlerweile besteht zumindest zwischen der Union, der SPD, der FDP und den Grünen ein Konsens über die Notwendigkeit eines stärkeren Wettbewerbs, um die Finanzierbarkeit des Gesundheitswesens sicherzustellen. Darüber, wie dieser Wettbewerb nach Ansicht der jeweiligen Partei ausgestaltet werden müsste, gehen die unterschiedlichen Auffassungen jedoch auseinander.

\section{Wettbewerbsideen zur Reform der GKV}

In der gesundheitsökonomischen Literatur zu Wettbewerb in der GKV wird zwischen verschiedenen Wettbewerbsmärkten differenziert (Abb.1). Dabei ist der Wettbewerb jeweils unterschiedlich stark ausgeprägt, was vor allem auf politische Steuerungsversuche zurückzuführen ist. Lediglich auf dem Versicherungsmarkt existieren die Bedingungen für einen ausgeprägten Wettbewerb: Im Jahr 1992 wurden Versicherten mit dem Gesundheitsstrukturgesetz erstmals weitreichende Wechselmöglichkeiten ermöglicht. Allerdings gibt es auch hier Wege, diesen Wettbewerb beispielsweise durch die Ausweitung von Wahltarifen $\mathrm{zu}$ intensivieren.

Zum Behandlungsmarkt existieren wegen des in der GKV herrschenden Prinzips der freien Arztwahl keine unmittelbaren Wettbewerbsregelungen. Die zunehmende Spezialisierung der Leistungserbringer und die Steuerungsversuche auf dem Leistungsmarkt beeinflussen den Behandlungsmarkt allenfalls indirekt, ohne dass die freie Arztwahl davon unmittelbar berührt wird. Dies könnte sich mit der 
Abbildung 1: Wettbewerbsmärkte in der gesetzlichen Krankenversicherung

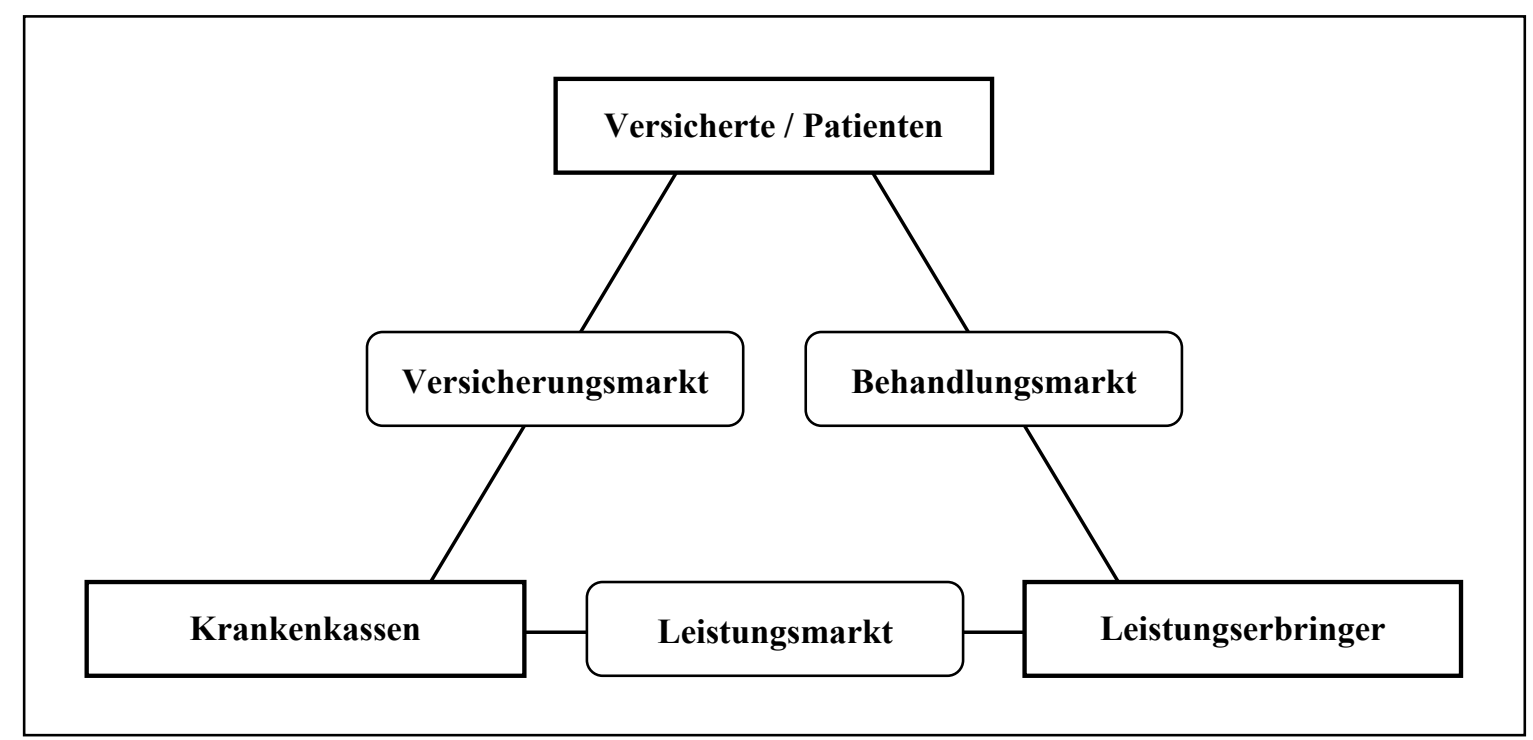

Ausweitung der bislang noch vergleichsweise schwach ausgeprägten Wettbewerbsstrukturen auf dem Leistungsmarkt ändern.

Insbesondere von einer stärkeren Ausrichtung des Leistungsmarktes auf Wettbewerb verspricht sich die Gesundheitsökonomie Einsparungen und Qualitätsverbesserungen in der GKV (Amelung 2007). Aufgrund der doppelten Facharztstruktur, die sich aus der sektoralen Trennung des deutschen Gesundheitswesens in ambulanten und stationären Bereich ergibt, werden große Ineffizienzen vermutet. Durch eine Integration der Versorgungsstrukturen könnten - so die Überzeugung der Ökonomen - nicht nur Einsparungen sondern auch Qualitätsverbesserungen erzielt werden. Aber auch mit Blick auf sektorinterne Strukturen hofft man auf Effizienzreserven. Beispielsweise könnten mit Hilfe des Hausarztes als Lotsen unnötige Doppeluntersuchungen vermieden werden. Stimuliert werden soll dieser Wettbewerb durch die gezielte Förderung von Selektivverträgen zwischen den Krankenkassen und den Leistungserbringern. Im Wettbewerb um den besten Vertrag würde auch auf Seiten der Leistungserbringer der Anreiz bestehen, größere Effizienzanstrengungen vorzunehmen, so die Annahme.

Je nach Wettbewerbsart entstehen unterschiedliche Anreize, die mal mehr, mal weniger erwünscht sind. Preise, Mengen oder Qualität können die Grundlage für Wettbewerb bilden. Zunächst ist dabei vorstellbar, dass Krankenkassen ausschließlich um den Preis konkurrieren. Sie versuchen, Einsparungen im Wettbewerb um Versicherte oder auf dem Leistungsmarkt zu erzielen. Hier besteht jedoch der Anreiz, die Qualität und Mengen der Leistungen zu reduzieren, um gesunkene Preise zu kompensieren. Daneben existiert die Möglichkeit, die Krankenkassen um die Qualität ihrer Leistungen konkurrieren zu lassen. Mit der Individualisierung des Krankenkassenangebotes sind allerdings Preiserhöhungen zu erwarten. Schließlich können Krankenkassen über Mengen, also die Quantität ihres Angebots, konkurrieren. Wegen des Anreizes zu Mengenausweitungen von Versicherten und Leistungserbringern sind Preissteigerungen bereits absehbar.

Um Fehlanreize durch eine bestimmte Wettbewerbsart so weit wie möglich zu begrenzen, bestehen in der GKV nebeneinander Regelungen zur Preissteuerung, zur Qualitätssicherung und zum Leistungskatalog. Von einer Dominanz einer dieser Wettbewerbsarten kann nicht gesprochen werden. In der politischen Debatte existieren unterschiedliche Zielvorstellungen hinsichtlich der Frage, welche Art von Wettbewerb auf welchem Markt stattfinden soll. Diese Ideen werden vor allem deshalb intensiv diskutiert, weil unmittelbar mit ihnen politische Überzeugungen verknüpft sind.

\section{Parteipolitische Wettbewerbsinteressen}

Die ersten Vorschläge zur Einführung von Wettbewerb in das GKV-System lagen bereits relativ früh im politischen Raum vor. Schon 1987 kritisierte die Enquete-Kommission „Strukturreform der Gesetzlichen Krankenversicherung“ unzureichende Wechselmöglichkeiten zwischen den Krankenkassen und mangelnde Effizienz in der Leistungserbringung (Enquete-Kommission 1990). Betont wurde vor allem, dass die korporatistische Steuerung deutliche Schwachstellen aufweist. Anstelle von Kostendämpfungsmaßnahmen auf Makro-Ebene sei das Ziel einer einnahmeorientierten Ausgabenpolitik besser durch die Ausweitung von Anreizen auf der Mikro-Ebene zu erreichen. Wettbewerb schien in diesem Zusammenhang ein geeignetes Mit- 


\section{THEMA}

tel zu sein, eben diese Anreize auf dem Versicherten- und dem Leistungsmarkt zu schaffen.

Dennoch setzte die von Helmut Kohl geführte schwarzgelbe Bundesregierung beim Gesundheitsreformgesetz von 1988 weiterhin auf altbewährte Mittel wie Globalbudgets und Zuzahlungen. Allerdings wurden diese Vorschläge mit einer Flut von Änderungsanträgen im Gesetzgebungsprozess seitens der Opposition und der Verbände durchlöchert. Erst nachdem die vom Kanzler selbst als „Jahrhundertwerk “ titulierte Reform wegen des erfolgreichen Widerstands als gescheitert galt, wurden Wettbewerbsideen tatsächlich als Lösungen zur Kostendämpfung attraktiv.

\section{Wettbewerb auf dem Versicherungsmarkt (1992-1998)}

1992 erklärte sich der damalige Gesundheitsminister Horst Seehofer - anders als seine Vorgänger - zu Gesprächen mit der Opposition bereit, um die Erfahrungen des Gesundheitsreformgesetzes nicht zu wiederholen. Nur wenige Wochen nach seinem Amtsantritt traf sich Seehofer mit Vertreter der Union, FDP und der SPD im rheinischen Lahnstein, wo unter Ausschluss der Öffentlichkeit über die Inhalte des Gesundheitsstrukturgesetzes (GSG) verhandelt wurde. Ein solcher Kompromiss sollte die Abwehr von Verbandsinteressen im Reformprozess und gleichzeitig die bereits angekündigte Blockade der Opposition im Bundesrat verhindern. Das Ergebnis der Verhandlungen war der parteienübergreifende Lahnstein-Kompromiss, mit dem weitreichende Strukturveränderungen und Einsparungen erzielt werden konnten. Mit dem gemeinsam ausgehandelten Entwurf schien aus den Fehlern gelernt worden zu sein.

Allerdings musste die Bundesregierung im Gegenzug deutliche Zugeständnisse hinnehmen. So brachte die SPD Regelungen zur Einführung von Wettbewerb auf dem Krankenkassenmarkt ab 1996 in den Gesetzentwurf ein. Zwar bestanden bereits für einen kleinen Teil der Versicherten bestimmte Wahlmöglichkeiten, für die Krankenkassen jedoch bedeutete diese Ausweitung der Wahlmöglichkeiten erhebliche Veränderungen. Über einen Risikostrukturausgleich sollten die unterschiedlichen Ausgangsbedingungen der Kassen in diesem Wettbewerb aufgrund einer unvorteilhaften Versichertenstruktur abgemildert werden. Die parallele Neuregelung von Kassenfusionen innerhalb einer Kassenart und von Schließungen unwirtschaftlicher Krankenkassen sorgte für zusätzliche Wettbewerbsanreize. Das diesen Regelungen zugrundeliegende Leitbild war die Vorstellung eines solidarischen Gesundheitswesens. Aus der Sicht der SPD ließ sich die Zuweisung von Versicherten zu bestimmten Kassenarten wegen der zum Teil großen Einkommensunterschiede zwischen den Versichertengruppen und der unterschiedlichen Versichertenstrukturen nicht mehr sachlich begründen. Ein soldiarischer Kassenwettbewerb erschien in diesem Zusammenhang als geeignete Lösung, die Finanzierungsprobleme einzelner Kassenarten zu lösen. Umgekehrt musste die SPD dagegen den Forderungen der FDP und Union nach mehr Zuzahlungen des Patienten zustimmten. Auch die FDP-Forderung zur Einführung eines Tarifs zur Kostenerstattung nach dem Vorbild der privaten Krankenversicherung fand Eingang in das Gesetz.

Angesicht dieser eigentlich widersprüchlichen Elemente wird deutlich, dass der Gesetzgeber keine einheitliche Regelungsphilosophie verfolgte, sondern sich vielmehr ein radikaler Wandel ohne kohärentes Steuerungsziel vollzog (Gerlinger 2002). Dennoch läutete das GSG einen Strategiewandel bei der Steuerung der GKV ein. Anstelle der korporatistischen und damit kollektivvertraglichen Regelungen wurden nunmehr verstärkt wettbewerbliche Regelungen gefördert. Der Trend in der Gesundheitspolitik ging damit weg vom „gemeinsam und einheitlich“ der Verbände hin zu Regelungen auf Marktebene. Dass ein derart weitreichender Wandel der GKV überhaupt durchgesetzt werden konnte, lag vor allem an dem bis dahin gewachsenen Einfluss der Verbände. Auch auf Seiten der Union setzte sich diese Erkenntnis ansatzweise durch. Die FDP hingegen musste diese Änderungen auch gegen ihren Willen hinnehmen, setzte im Gegenzug aber die Einführung eines Kostenerstattungstarifs für freiwillig Versicherte in der GKV durch. Die steuerungspolitischen Veränderungen sind somit nicht generell auf Lernprozesse aller politischen Akteure zurückzuführen, sondern resultieren eher aus dem Kompromisszwang im föderalen Regierungssystem.

Die ursprünglich beabsichtigte Stabilisierung der Beitragssätze konnte allerdings nicht dauerhaft erreicht werden. Wegen der konjunkturellen Entwicklung, der steigenden Arbeitslosigkeit und der auslaufenden Budgetierungen war ein steigender Kostendruck absehbar. Somit entstand schon kurz nach der Bundestagswahl 1994 aus Sicht der fortgesetzten konservativ-liberalen Regierungskoalition wieder ein dringender Handlungsbedarf. Die SPD plante, auch den Leistungsmarkt für wettbewerbliche Elemente zu öffnen. Im Gegensatz zu den Verhandlungen im Vorfeld des GSG schloss die FDP allerdings aus, wie beim Lahnstein-Kompromiss zusammen von Union und SPD übergangen zu werden, und ließ dies auch in der Koalitionsvereinbarung festhalten (Reiners 2008). Nachdem bei Seehofer die kurzzeitige Hoffnung bestand, einen parteiübergreifenden Kompromiss zu wiederholen, mussten diese Reformpläne also aufgrund der Absage der FDP und der drohenden Blockade der SPD im Bundesrat auf nicht zustimmungspflichte Regelungen beschränkt werden. Die beiden GKV-Neuordnungsgesetze wurden schließlich 1997 beschlossen. Im zweiten Neuordnungsgesetz (2. GKVNOG) wurde auch der Kassenwettbewerb weiter ausgebaut.

Die Krankenkassen erhielten hier Möglichkeiten, ihre Angebote im Wettbewerb voneinander zu differenzieren. So wurde die Wahl eines Kostenerstattungstarifs für alle Versicherten freigestellt und den Kassen der Abschluss von Tarifen mit Selbstbehalten für freiwillig Versicherte zugestanden. Zudem sollten Kassen ihren Versicherten Beitragsrückzahlungen gewähren können, wenn in einem Kalenderjahr keine Leistungen in Anspruch genommen wurden. Für über den Leistungskatalog hinausgehende Leistungen konnten die Kassen Zuzahlungen einführen. Schließlich erhielten die Kassen das Recht, erweiterte Leistungen, wie präventive Maßnahmen, Kuren, Rehabilitatio- 
nen und Hilfsmittel anzubieten. Diese Maßnahmen zielten vor allem auf den Preis des Kassenangebots. Verglichen mit der SPD verfolgten Union und FDP somit andere Interessen beim Wettbewerb auf dem Versicherungsmarkt. Während seitens der SPD der Kassenwettbewerb als Instrument betrachtet wurde, die GKV solidarischer zu gestalten, zielte die Regierungskoalition um auf eine größere Wirtschaftlichkeit bei den Krankenkassen führen, ohne dass dabei die Strukturen der Leistungserbringer beeinflusst werden. Leitend waren hier die Überzeugung ineffizienter Krankenkassen mit hohen Verwaltungskosten und der Notwendigkeit einer uneingeschränkten Arztwahl. Nach dem Regierungswechsel von 1998 konnte schließlich die SPD umgekehrt ihre Wettbewerbsvorstellungen in Reformen umsetzen.

\section{Wettbewerb auf dem Leistungsmarkt (1998-2005)}

Bereits in ihrem Koalitionsvertrag legte die neue rotgrüne Regierung das weitere Vorgehen in der Gesundheitspolitik fest (SPD/Bündnis 90/Die Grünen 1998). So sollte noch 1998 ein Vorschaltgesetz verabschiedet werden, das die Voraussetzungen für eine durchgreifende Strukturreform ab dem Jahr 2000 schaffen sollte. Ziel war es, die Versorgungsstrukturen des Gesundheitswesens mit expliziter Betonung des Solidar- und Sachleistungsprinzips in Richtung einer höheren Qualität und größerer Effizienz umzubauen. Mit dem Vorschaltgesetz sollte eine vorläufige Ausgabenbegrenzung stattfinden sowie die von schwarz-gelb beschlossenen Zuzahlungen und Elemente der privaten Krankenversicherung (PKV) zurückgeführt werden. Die daran anschließende Strukturreform sollte für „mehr Wettbewerb um Qualität, Wirtschaftlichkeit und effizientere Versorgungsstrukturen "sorgen, indem ein Globalbudget eingeführt und die Rolle der Hausärzte und die Integration der Sektoren verbessert werden.

Besetzt wurde das Ressort Gesundheit damals überraschend mit der relativ jungen und in der Gesundheitspolitik eigentlich unerfahrenen Grünen-Politikerin Andrea Fischer. Auf der Grundlage des Koalitionsvertrags erarbeitete Fischer das GKV-Solidaritätsstärkungsgesetz (GKV-SolG) als Vorschaltgesetz. Mit Blick auf die Wettbewerbsregelungen wurde die Ausweitung des Kostenerstattungstarifs auf alle Versicherten zurückgenommen. Gegen die vollständige Abschaffung sprach aus der Sicht von Fischer, dass die GKV dieses Instrument im Wettbewerb mit der PKV um freiwillig Versicherte nutzen konnte. Der Tarif zu Selbstbehalten konnte analog nur von freiwillig Versicherten genutzt werden. Trotz der ursprünglichen Kritik an den von der Vorgängerregierung eingeführten Tarifen zu Beitragsrückzahlungen und Zuzahlungen wurden diese Regelungen beibehalten. Kassen mit einer hohen Zahl von chronisch Kranken sollten diese Tarife nutzen dürfen, um ihre Finanzierung zu sichern. Die Beitragsrückzahlungen sollten den Kassen gleichzeitig als ein Instrument im Wettbewerb um besondere Versorgungsformen dienen. Zudem erhielt der Versicherte ein einmonatiges Sonderkündigungsrecht, für den Fall dass seine Krankenkasse ihren Beitragssatz anhebt. Dies bedeutete eine deutliche
Verschärfung des Preiswettbewerbs, die jedoch nicht unumstritten mitgetragen und insbesondere von Sozialpolitikern der SPD kritisiert wurde. Dennoch konnte sich das von den Grünen geführte Bundesministerium für Gesundheit mit seinen Vorstellungen gegen den Koalitionspartner durchsetzen.

Als die Opposition bereits 1999 die Mehrheit im Bundesrat zurückgewann, waren SPD und Grüne früher als erwartet auf Kompromisse angewiesen. Dennoch wollte die Regierung nicht vom Ziel einer Neuordnung der Versorgungsstrukturen abrücken und formulierte wegen der Blockade des Bundesrats nur nicht zustimmungspflichtige Maßnahmen beim GKV-Gesundheitsreformgesetz 2000 (GRG-2000). Im Ergebnis zielte die Reform auf eine bessere Verzahnung von ambulanter und stationärer Versorgung.

Die SPD sah sich nun in der Lage, Regelungen umsetzen zu können, die sie schon in der vorangegangenen Legislaturperiode angestrebt hatte. So hatte sie bereits 1996 in der Opposition mit ihrem Entwurf zum GSG II versucht, den Kassen größere Spielräume zu Selektivverträgen mit Leistungserbringern einzuräumen. Zwar griffen Union und FDP einige der Vorschläge auf, verfolgten mit ihnen allerdings andere Ziele. Bereits 1997 wurden Modellvorhaben eingeführt, die innovative Verfahren und Organisationsformen der Leistungserbringung in allen Sektoren zuließen. Anders als von der SPD beabsichtigt, orientierten sich diese Regelungen allerdings explizit am Kollektivvertragssystem. Zwar sollten Wirtschaftlichkeitsreserven genutzt werden, dabei verblieb die Initiative zum Abschluss solcher Verträge jedoch beim ambulant tätigen Arzt. Dieser allein sollte entscheiden können, ob er Kooperationen eingehen will. Vor allem sollten Gruppen von Ärzten und Zahnärzten erweiterte Kooperationsmöglichkeiten in Ballungszentren gegeben werden. Für die SPD war es im Zuge des GRG-2000 vergleichsweise leicht, die Modellvorhaben zu Selektivvertragsregelungen umzugestalten. Nun waren die Kassenärztlichen Vereinigungen nicht mehr exklusive Vertragspartner, sondern Kassen konnten Verträge mit einzelnen oder Gruppen von Leistungserbringern abschließen.

Daneben wurde die hausarztzentrierte Versorgung eingeführt. Vor allem die SPD erhoffte sich hiervon, dass die freiwillig teilnehmenden Versicherten von einer besseren Versorgung profitieren, weil mit der Patientensteuerung durch den Hausarzt - sozusagen als Lotsen im GKV-System - die Zahl unnötiger Untersuchungen reduziert wird. Ursprünglich plante die SPD einen Systemwechsel in Richtung eines Primärarztsystems, bei dem Hausärzte verpflichtend zur ersten Anlaufstelle bei der Behandlung geworden wären. Allerdings musste diese Regelungen fallengelassen werden, weil damit das gesamte GRG-2000 aufgrund der damit notwendigen Zustimmungspflicht vom Bundesrat abgelehnt worden wäre.

Eingeführt wurde auch die integrierte Versorgung, bei der die Krankenkassen erstmalig Verträge mit Gemeinschaften von Leistungserbringern und ohne die Einbindung der Kassenärztlichen Vereinigungen über eine sektorenübergreifende Versorgung abschließen durften. Für den ambulanten Bereich war allerdings eine Rahmen- 


\section{THEMA}

vereinbarung der Krankenkassen- und Ärzteverbände notwendig. Um zu vermeiden, dass diese Modelle über die bestehende Versorgung hinaus finanziert werden, sollte die Gesamtvergütung um den Anteil bereinigt werden, den diese Versorgungsverträge im Kollektivvertragssystem verursacht hätten.

Zusammenfassend wurden Regelungen getroffen, die zum ersten Mal den Wettbewerb auf dem Leistungsmarkt betrafen. Vor allem der SPD gelang es, ihre Überzeugungen hinsichtlich der effizienz- und qualitätssteigernden Wirkungen eines Wettbewerbs zwischen den Leistungserbringern auch gegen den Widerstand der Ärzteschaft umzusetzen. Trotz der Blockade der Opposition im Bundesrat konnte das Gesetz nicht gestoppt werden.

Nach der Bundestagswahl 2002 vereinbarte die Regierungskoalition die Fortsetzung dieser am Leistungsmarktwettbewerb orientierten Politik (SPD/Bündnis 90/Die Grünen 2002). Bundesgesundheitsministerin Ulla Schmidt, die 2001 die Leitung des Ressorts übernahm, erarbeitete einen Entwurf zum Gesundheitsmodernisierungsgesetz (GMG). In seiner ersten Fassung enthielt der Reformentwurf einige äußert radikale Strukturelemente. So war zunächst geplant, dass der Sicherstellungsauftrag der ambulanten Facharzt- und Krankenhausversorgung auf die Krankenkassen übergehen soll. Damit hätten die Kassen gegenüber den Leistungserbringern deutlich an Einfluss gewonnen. Aus wettbewerblicher Sicht hätte diese Regelung den größten Einschnitt in der Neuordnung der Versorgung dargestellt. Nach zähen Verhandlungen in einer Arbeitsgruppe unter Beteiligung der Union wurde der Gesetzentwurf überarbeitet. Der Reformfokus lag noch immer auf der Weiterentwicklung der Versorgungsstrukturen, jedoch konnte die Union die Pläne zum Sicherstellungsauftrag abwenden, da sonst die flächendeckende Versorgung gefährdet und die freie Arztwahl eingeschränkt würde. Darüber hinaus wurde auf Initiative der Union der Tarif zur Kostenerstattung wieder aufgenommen. Die Regierungskoalition konnte sich dagegen mit einer Reihe von Regelungen zum Leistungsmarkt durchsetzen.

Bei der hausarztzentrierten Versorgung wurden die Krankenkassen nun verpflichtet, Verträge mit einzelnen oder Gemeinschaften von Hausärzten abzuschließen. Das schleppende Anlaufen dieser Verträge sollte so beschleunigt werden. Bei der integrierten Versorgung reagierte die Regierung auf die Möglichkeiten der Ärzteverbände, die Vertragsabschlüsse weitgehend blockieren konnten. Geändert wurde hier deshalb, dass die Kassenärztlichen Vereinigungen ausdrücklich nicht mehr als Vertragspartner in Frage kamen. Auch die Einführung einer bis 2006 befristeten Anschubfinanzierung, die die schwer umsetzbare Bereinigungsregelung ablöste, sollte den Kassen Anreize zum Abschluss dieser Verträge bieten. Mit den neuen Regelungen zu Medizinischen Versorgungszentren sollte zudem die Integration der Versorgungsstrukturen vorangetrieben werden. Die Krankenkassen sollten auch hier selektive Verträge abschließen können.

Hinsichtlich des Wettbewerbs auf dem Leistungsmarkt machte die Union also deutliche Zugeständnisse gegen- über der Bundesregierung, um den Übergang des Sicherstellungsauftrags an die Krankenkassen zu verhindern. Mit dieser Reform somit wurde die Machtverschiebung zugunsten der Finanzierungsträger fortgesetzt und die wettbewerblichen Steuerungsinstrumente ausgeweitet. Damit stellt die zweite Sachkoalition aus Union und SPD nach dem GSG keinen Bruch mit den bisherigen Steuerungsmechanismen dar. Allerdings schien sich das GKV-System an einem Scheideweg zu befinden, an dem die Union auf die Verlagerung der Kosten für Gesundheit auf den Versicherten favorisierte, während die SPD den tiefgreifenden Umbau der Versorgungsstrukturen beabsichtigte.

In der Tat knüpften Union und SPD diese Systemfrage an die Wahlentscheidung des Bürgers bei der nächsten Bundestagswahl. Nachdem die Rürup- und die HerzogKommission jeweils ihre Abschlussberichte vorgelegt hatten, kristallisierte sich in innerparteilichen Diskursen heraus, dass die SPD mit der Bürgerversicherung und die Union mit den Gesundheitsprämien in den Wahlkampf gehen wollte. Der Intention der SPD eines stärkeren Wettbewerbs in einem solidarischeren System stand der Anspruch der CDU zu einer nachhaltigen Finanzierbarkeit gegenüber. Erst nachdem sich keine der Parteien in der vorgezogenen Bundestagswahl durchsetzen konnte, begann mit der Bildung der Großen Koalition der Prozess zu einer gemeinsamen Problemdefinition. Die Grundlage für einen parteiübergreifenden Kompromiss der neuen Koalition bildete die Wettbewerbsstrategie.

\section{Konkurrierende Wettbewerbsvorstellungen (2005-2009)}

Hinsichtlich der Parteienkonstellation stellt die Große Koalition eine Besonderheit dar, denn im Gegensatz zu den davorliegenden Reformen erhoben sowohl Union als auch SPD gleichermaßen den Anspruch, ihre Interessen gegenüber dem Koalitionspartner durchzusetzen. Während in anderen Regierungskonstellationen unter der Kompromissnotwendigkeit von Zustimmungsgesetzen bei durchgreifenden Strukturreformen zwar eine große Sachkoalition vorherrschte, war diese aber immer durch die Dominanz der jeweiligen Regierungspartei im Agenda-Setting bestimmt. Nun stellte bereits die Formulierung des Koalitionsvertrags eine erste Hürde dar, weil Union und SPD versuchtem, ihre eigenen Forderungen in die Vereinbarung aufzunehmen und gleichzeitig die Forderungen des Koalitionspartners zu verhindern. Strittig waren vor allem Formulierungen, die auf eine Einführung von Kopfpauschale oder Bürgerversicherung deuteten. Im Ergebnis blieb der Koalitionsvertrag äußerst vage in Bezug auf Finanzierungsfragen. Weil bereits vor der Bundestagswahl zwischen Union und SPD Einigkeit über die mangelnde Effizienz im GKV-System herrschte, fiel die besondere Betonung des Wettbewerbs relativ leicht (CDU/ CSU/SPD 2005). Die Forderungen der Union und der SPD berührten einander nicht direkt, weil sie unterschiedliche Wettbewerbsmärkte betrafen, und konnten so parallel in Koalitionsvertag aufgenommen werden. Es wurden wenig konkrete Maßnahmen vereinbart, sondern lediglich, dass 
die Kassen und Leistungserbringer in größerem Ausmaß über Umfang, Preise und Qualität verhandeln dürfen sollten, ohne dass der Sicherstellungsauftrag der Kassenärztlichen Vereinigungen ausgehöhlt wird.

Die Diskussion über die konkrete Ausgestaltung einer Gesundheitsreform erfolgte in einer im April 2006 eingesetzten Koalitionsarbeitsgruppe von Fachpolitikern unter der Leitung von Ulla Schmidt. Die Konfliktlinien zwischen den Koalitionsparteien waren bei den Finanzierungsfragen jedoch so groß, dass eine Schlichtung auf regelmäßigen Treffen der Parteispitzen vorgenommen werden mussten.

Im Juli 2006 einigten sich die Spitzen von Union und SPD auf ein gemeinsames Eckpunktepapier zum GKVWettbewerbsstärkungsgesetz (GKV-WSG), in dem erstmalig der Kompromissvorschlag des Gesundheitsfonds zu den Modellen von Bürgerversicherung und Kopfpauschale formuliert wurde (CDU/CSU/SPD 2006). Dabei hatte der Gesundheitsfonds an sich weniger eine wettbewerbsordnende Funktion, als dass er die Organisationsstrukturen der GKV-Finanzierung neuordnete. Auch wenn die Union zunächst die Abkopplung der Krankenversicherungsbeiträge von den Lohnnebenkosten forderte, blieb es bei der Finanzierung aus prozentualen Beitragssätzen, die sich am Einkommen bemessen. Die Arbeitgeber- und Arbeitnehmerbeiträge sollten von Krankenkassen eingezogen und an den Gesundheitsfonds weitergeleitet werden. Allerdings war neu, dass der Preiswettbewerb zwischen den Kassen nicht über unterschiedliche Beitragssätze stattfinden sollte. Mit der Einführung des Fonds wurde ein bundesweit einheitlicher Beitragssatz eingeführt. Im ersten Jahr sollte dieser die gesamten Kosten der Kassen decken, in den darauffolgenden Jahren allerdings nur 95 Prozent aller Ausgaben tragen. Die Kassen sind damit in Zukunft zu höherer Wirtschaftlichkeit und kontinuierlich zu Einsparungen gezwungen. Für den Fall, dass sie ihre Ausgaben nicht decken können, dürfen sie kassenindividuelle Zusatzbeiträge von ihren Mitgliedern erheben. Aus Sicht der SPD wurde der Preiswettbewerb auf dem Versicherungsmarkt wurde damit begrenzt, denn dieser beschränkt sich nunmehr lediglich auf den Zusatzbeitrag. Für die Union war dieser Kompromiss akzeptabel, weil sie mit ihm den Einstieg in die Trennung von Gesundheits- und Lohnnebenkosten geschafft hatte.

Darüber hinaus wurde der Wettbewerb um effiziente Versorgungsstrukturen gestärkt. So wurden die bestehenden Wahltarife auf eine einheitliche Regelungsgrundlage gestellt und für Versicherte eine Mindestvertragsbindung von drei Jahren eingeführt. Die Verpflichtung bei der Teilnahme an besonderen Versorgungsformen ist lediglich auf ein Jahr beschränkt, um dadurch den Wettbewerb zu fördern. Zunächst wollte die Union die generelle Ablösung des Sachleistungsprinzips durch das Kostenerstattungsprinzip, konnte sich jedoch nicht gegen die SPD durchsetzen. Dagegen konnte die SPD ihre Forderung durchbringen, den ursprünglichen Kerngedanken von Modellvorhaben und Strukturverträgen vollständig aufzugeben und sie gänzlich selektivvertraglich auszurichten.
Bei der hausarztzentrierten Versorgung erfolgte ebenfalls eine stärkere einzelvertragliche Orientierung, indem die Kassenärztlichen Vereinigungen mit der Einwilligung der Hausärzte einbezogen werden durften. Die Union konnte hingegen wieder einmal verhindern, dass das von der SPD in der Vergangenheit immer wieder geforderte Primärarztsystem eingeführt wurde, denn die Teilnahme des Versicherten an der hausarztzentrierten Versorgung blieb freiwillig. Daneben setzte sie durch, dass Hausarztverträge nicht quersubventioniert werden dürfen.

Bei der integrierten Versorgung sollte dagegen eine stärkere populationsbezogene Versorgung gefördert werden. Bislang konzentrierten sich die Verträge auf eine sektorenübergreifende Versorgung von nur einer Volkskrankheit. Eine nochmalige Verlängerung der inzwischen bis Ende 2008 laufenden Anschubfinanzierung war nicht mehr mit der Union auszuhandeln, denn sie argumentierte, dass sich die Verträge von nun an selbst finanzieren müssten. Aus ihrer Sicht kamen die bestehenden Verträge nur wegen der zusätzlichen Finanzierung zustande und boten keine Effizienzverbesserungen.

Auch wenn Union und SPD informell unter Ausschluss der Öffentlichkeit und begleitet von der lauten Kritik der Verbände verhandelten (Knieps 2007), gelang ihnen nicht der im Wahlkampf angekündigte große Wurf. Dies lag vor allem an der Konstellation in der Großen Koalition, in der keine der beiden Parteien das Agenda-Setting im Gesetzgebungsprozess dominierte. Zwar konnten weder Union noch SPD ihre vor der Bundestagswahl formulierten Modelle umsetzen, jedoch konnten sie Teilerfolge erzielen. Dabei hat vor allem die SPD ihre Ziele gegenüber der Union durchsetzen können, indem mit den wettbewerblichen Regelungen die Weiterentwicklung der Versorgungsstrukturen fortgeführt und somit die Qualität - und weniger der Preis - der Leistungen in den Vordergrund gerückt wurden.

Mit dem Gesetz zur Weiterentwicklung der Organisationsstrukturen (GKV-OrgWG) von 2008 setzte die Große Koalition ihre am Wettbewerb orientierte Politik fort. Auch hier konnten die Koalitionsparteien jeweils nur Teilerfolge erzielen. Zunächst sollte das GKV-OrgWG die Krankenkassenstrukturen für den verschärften Wettbewerb anpassen und das Insolvenzrecht für alle Kassen anwendbar machen. Geregelt wurden außerdem die Haftungsansprüche bei Insolvenzen und kassenartinterne Finanzhilfen zur Verhinderung von Insolvenzen und Schließungen. Damit konnte sich die SPD mit ihrer Vorstellung eines solidarischen Wettbewerbs durchsetzten. Die neueingeführte Verpflichtung der Kassen zum Abschluss von Verträgen mit Gemeinschaften von Hausärzten dagegen gilt als Zugeständnis gegenüber der Union. Selektivverträge zur hausarztzentrierten Versorgung sind nur noch über diese Verträge hinaus möglich. Da die Kassen wenig Anreize für eine solche Doppelversorgung mit Hausarztverträgen haben, wurde der Wettbewerb zwischen den Leistungserbringern somit faktisch wieder eingeschränkt. 


\section{THEMA}

\section{Fazit}

Wie bei der Untersuchung des Einführungsprozesses von Wettbewerbsstrukturen in das deutsche GKV-System deutlich wird, sind Veränderungen in diesem Politikfeld wegen der Vielzahl von Akteuren mit Einflussmöglichkeiten nur über einen langen Zeitraum möglich. Ob sich Wettbewerb mittlerweile tatsächlich etabliert hat, hängt zentral von den jeweiligen Vorstellungen über die Ausgestaltung und das Ausmaß eines solchen Wettbewerbs ab. So hatten die unterschiedlichen Akteure „kognitive Blaupausen“, anhand derer Reformnotwendigkeiten des bestehenden Systems bewertet wurden. Aus der jeweiligen Wahrnehmung gab es und gibt es immer noch entweder zu wenig, zu viel oder den falschen Wettbewerb.

Trotz der deutlichen Zunahme von wettbewerblichen Steuerungsinstrumenten im ambulanten Sektor kann nicht uneingeschränkt von der Entmachtung korporatistischer Strukturen und einer Ausrichtung der GKV auf Wettbewerb gesprochen werden (Gerlinger 2010). Angesichts des unsicheren Wissens über den Outcome politischer Steuerung kann allerdings keiner der politischen Akteure mit großer Sicherheit prognostizieren, ob Wettbewerbsregelungen überhaupt die erwarteten Wirkungen erzielen. Politik kann lediglich indirekt mit Hilfe rechtlicher Regelungen steuern. Hierarchische Steuerungsversuche entfalten ihre Wirkung jedoch häufig nur kurzzeitig und zwar solange, bis Akteure neue Wege finden, ihre Interessen anderweitig durchzusetzen.

Mit der Einführung von Wettbewerbselementen wird allerdings die Problematik indirekter Steuerung verschärft. So nimmt die Anzahl der Akteure im Zuge der Verlagerung der Steuerungskompetenzen auf die Mikro-Ebene deutlich zu. Während noch im vorrangig korporatistischen Gesundheitssystem wenige Akteure für die Politik als Steuerungsadressaten in Frage kamen, haben sich die Interessen mit der Zuwendung hin zu wettbewerblichen Instrumenten erheblich ausdifferenziert. Damit ergeben sich zugleich neue Steuerungserfordernisse. Der Patient muss im Wettbewerb in der Lage sein, bei der Wahl seiner Krankenkasse auf transparente Qualitätskriterien zurückgreifen zu können, sonst ließe sich eine effizientere Behandlung nicht gewährleisten. Der Spitzenverband Bund der gesetzlichen Krankenkassen soll deshalb Rahmenrichtlinien für Qualitätsund Leistungsbenchmarks der Krankenkassen beschließen (Stackelberg/Weller 2008). Auch bei der Leistungserbringung werden kontinuierlich Instrumente zur Qualitätsbewertung und Qualitätssicherung eingeführt und ausgebaut (Sauerland 2009). Damit hat die Einführung des Wettbewerbs insgesamt zu einem Bedeutungswandel der korporatistischen Steuerung geführt. Mit diesem Bedeutungswandel geht ein Steuerungsverlust durch die abnehmende Verpflichtungsfähigkeit der Akteure aus der Mikro-Ebene einher (Bandelow 2009).

Bei der Betrachtung der Wettbewerbsregelungen zum Versicherten- und Leistungsmarkt fällt zudem auf, dass sich bei den großen Strukturreformen des GSG von 1992 und dem GMG von 2003 die jeweilige Regierungskoalition in der Sachkoalition mit ihren Wettbewerbsvorstellungen durchsetzen konnte. Beim GKV-Wettbewerbsstärkungsgesetz mit der gleichgestellten Beteiligung von Union und SPD gab es zwar Regelungen auf beiden Wettbewerbsmärkten, doch hatte die SPD mit der Leitung des Bundesministeriums für Gesundheit und der langjährigen Erfahrung der Ministerin Ulla Schmidt einen strategischen Vorteil. Daraus kann geschlossen werden, dass der "große Wurf“ vorausgesetzt, er ist überhaupt möglich (Altenstetter/Busse 2005) - nur dann gelingen kann, wenn sich die Parteien der SPD und Union in einer Großen (Sach-)Koalition auf eine Reform einigen. Während der Verhandlungen dominieren die Parteiinteressen in der Regel wegen des Zwangs zu vorzeigbaren Ergebnissen. Zwar gab es jeweils Vorstöße von nur SPD oder Union, die aber wegen der Zustimmungspflicht des Bundesrats nicht durchsetzbar waren. Daher musste der Kompromiss in Verhandlungen gesucht werden. Konfrontative und kompetitive Regelungsversuche blieben hinter den eigentlichen Erwartungen zurück. Auch wenn diese Kompromisse einschneidende Regelungen zum Wettbewerb in der GKV hervorgebracht haben, konnte die Politik nicht unmittelbar steuernd in den Umsetzungsprozess eingreifen. Stattdessen steuerte sie immer wieder durch die Neufassung oder Änderung gesetzlicher Regelungen nach, in der Hoffnung dass ihre Interessen von den Akteuren umgesetzt werden. Von einem radikalen Wandel kann deshalb eigentlich nur bei der Einführung des Kassenwettbewerbs gesprochen werden, bei dem den Akteuren kein Spielraum bei der Umsetzung zugestanden wurde.

Mit Blick auf die unterschiedlichen Regelungen zum Wettbewerb - nicht nur im ambulanten Bereich sondern auch in den anderen Sektoren - wird deshalb häufig ein „Steuerungswirrwarr“ (Cassel et al. 2006) beklagt. Dabei wird allerdings oft vergessen, dass sich die Vielzahl von Regelungen aus den unterschiedlichen parteipoltischen Vorstellungen über die Ausgestaltung dieses Wettbewerbs ergibt. Diese sind nicht immer miteinander vereinbar, so dass aufgrund der Kompromissnotwendigkeit die eigentlichen Regelungsabsichten aufgeweicht werden und Inkonsistenzen entstehen. So versuchte die SPD den Wettbewerb um Qualität auf dem Leistungsmarkt zu fördern, die Union hingegen verfolgte das Ziel eines größeren Preiswettbewerbs auf dem Versicherungsmarkts. Dieses inkrementelle „Muddling-through“, das aus der Perspektive von Partialinteressen als ineffizient bewertet wird, erfüllt aus gesamtgesellschaftlicher Perspektive jedoch eine nicht zu unterschätzende Funktion. Bei aller Pluralität von Interessen muss sichergestellt sein, dass Politik unabhängig von Interessengruppen entscheiden kann. Nur so sind politische Akteure imstande, auch weniger organisations- und durchsetzungsfähige Interessen angemessen zu berücksichtigen.

\section{Literatur}

Altenstetter, Christa/Busse, Reinhard (2005): Health Care Reform in Germany: Patchwork Change within Established Governance Structures, in: Journal of Health Politics, Policy and Law 30/1-2, S. 121-142.

Amelung, Eric Marc (2007): Managed Care: Neue Wege im Gesundheitsmanagement, 4. Aufl., Wiesbaden: Gabler. 
Bandelow, Nils C. (2006): Gesundheitspolitik in der Bundesrepublik Deutschland: Zielkonflikte und Politikwechsel trotz Blockaden, in: Schmidt, Manfred G./Zohlnhöfer, Reimut (Hg.): Regieren in der Bundesrepublik Deutschland. Innen- und Außenpolitik seit 1949. Wiesbaden: VS Verlag für Sozialwissenschaften, S. 159-176.

Bandelow, Nils C. (2009): Health Governance in the Aftermath of Traditional Corporatism: One Small Step for the Legislator, One Giant Leap for the Subsystem?, in: German Policy Studies 5/1, S. 43-63.

Cassel, Dieter/Ebsen, Ingwer/Greß, Stefan/Jacobs, Klaus/Schulze, Sabine/ Wasem, Jürgen (2006): Weiterentwicklung des Vertragswettbewerbs in der gesetzlichen Krankenversicherung. Vorschläge für kurzfristig umsetzbare Reformschritte. Gutachten des AOK-Bundesverbandes.

CDU/CSU/SPD (2005): Gemeinsam für Deutschland. Mit Mut und Menschlichkeit. Koalitionsvertrag von CDU, CSU und SPD vom 11. November 2005. Rheinbach.

CDU/CSU/SPD (2006): Eckpunkte zu einer Gesundheitsreform 2006. 4. Juli 2005. Berlin.

Enquete-Kommission (1990): Endbericht der Enquete-Kommission „Strukturreform der gesetzlichen Krankenversicherung“. BT-Drs. 11/6380.

Gerlinger, Thomas (2002): Vom korporatistischen zum wettbewerblichen Ordnungsmodell? Über Kontinuität und Wandel politischer Steuerung im Gesundheitswesen, in: Gellner, Winand/Schön, Markus (Hg.): Paradigmenwechsel in der Gesundheitspolitik? Baden-Baden: Nomos, S. $123-151$.

Gerlinger, Thomas (2010): Health Care Reform in Germany, in: German Policy Studies 6/1, S. 107-142.

Knieps, Franz (2007): Hitler, Honecker und die Gesundheitsreform. Zur Entstehungsgeschichte des GKV Wettbewerbsstärkungsgesetzes, in: Ulrich, Volker/Walter, Ried (Hg.): Effizienz, Qualität und Nachhaltigkeit im
Gesundheitswesen. Theorie und Politik öffentlichen Handelns, insbesondere in der Krankenversicherung. Festschrift zum 65. Geburtstag von Eberhard Wille. Baden-Baden: Nomos, S. 871-879.

Reiners, Hartmut (2008): Die Bundesländer bei der Reform der GKV, in: Schroeder, Wolfgang/Paquet, Robert (Hg.): Gesundheitsreform 2007. Nach der Reform ist vor der Reform. Wiesbaden: VS Verlag für Sozialwissenschaften, S. 50-57.

Sauerland, Dirk (2009): The legal framework for health care quality assurance in Germany, in: Health Economics, Policy and Law 4, S. 79-98.

SPD/Bündnis 90/Die Grünen (1998): Aufbruch und Erneuerung - Deutschlands Weg ins 21. Jahrhundert. Koalitionsvereinbarung zwischen der Sozialdemokratischen Partei Deutschlands und Bündnis 90/Die Grünen vom 20. Oktober 1998. Bonn.

SPD/Bündnis 90/Die Grünen (2002): Erneuerung - Gerechtigkeit - Nachhaltigkeit. Für ein wirtschaftlich starkes, soziales und ökologisches Deutschland. Für eine lebendige Demokratie. Koalitionsvereinbarung zwischen der Sozialdemokratischen Partei Deutschlands und Bündnis 90/Die Grünen vom 16. November 2002. Berlin.

Stackelberg, Johann-M. von/Weller, Michael (2008): Der GKV-Spitzenverband als neuer Player im Gesundheitswesen, in: Repschläger, Uwe (Hg.): BARMER Gesundheitswesen aktuell 2008: Beiträge und Analysen zu Auswirkungen der Gesundheitsreform ab 2009, Wuppertal: Barmer Hauptverwaltung, S. 182-198.

Wasem, Jürgen (1999): Staatliche Steuerung des Gesundheitswesens - Historie und Effekte von Kostendämpfungsmaßnahmen und Gesundheitsreformen, in: Wille, Eberhard (Hg.): Zur Rolle des Wettbewerbs in der gesetzlichen Krankenversicherung. Gesundheitsversorgung zwischen staatlicher Administration, korporativer Koordination und marktwirtschaftlicher Steuerung, Baden-Baden: Nomos, S. 9-29.

\title{
Gesundheitsstandort privater Haushalt
}

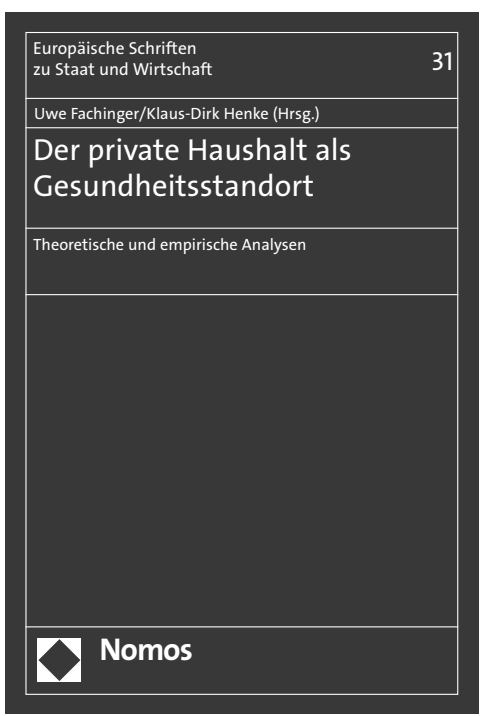

\author{
Der private Haushalt als Gesundheitsstandort \\ Theoretische und empirische Analysen \\ Herausgegeben von Univ. Prof. Dr. Uwe Fachinger und \\ Prof. Dr. Klaus-Dirk Henke \\ 2010, 240 S., brosch., 59,-€, ISBN 978-3-8329-5718-6 \\ (Europäische Schriften zu Staat und Wirtschaft, Bd. 31)
}

Der private Haushalt wird sich durch den zunehmenden Einsatz von assistierenden Technologien und Ambient Assisted Living-Systemen (AAL) als Gesundheitsstandort herausbilden. In den Beiträgen werden die damit verbundenen ökonomischen Aspekte insbesondere im Zusammenhang mit der gesundheitlichen und pflegerischen Versorgung älterer Menschen behandelt.

Bitte bestellen Sie im Buchhandel oder

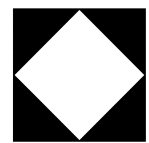

Nomos versandkostenfrei unter - www.nomos-shop.de 